\title{
A Study of Walking Speed Measurement for Elderly Health Assessment Using Ultrahigh-frequency Radio-frequency Identification Tags
}

\author{
Lingfei Mo, ${ }^{1 *}$ Chenyang Li, ${ }^{2}$ Hualin Huang, ${ }^{1}$ and Yaxuan Dong ${ }^{1}$ \\ ${ }^{1}$ School of Instrument Science and Engineering, Southeast University, \\ No. 2 Sipailou, Nanjing, Jiangsu, 210096, China \\ ${ }^{2}$ College of Engineering, Shanghai Polytechnic University, \\ No. 2360 Jinhai Road, Pudong, Shanghai, 201209, China
}

(Received January 11, 2018; accepted February 28, 2018)

Keywords: discrete generalized cross-correlation, Kalman filter, partitioned Gaussian fitting, UHF RFID, health assessment of the elderly, walking speed

The precise measurement of walking speed in daily life is essential for the health guidance and life prediction of the elderly. Ultrahigh frequency (UHF) radio frequency identification (RFID) tags have the advantages of compact size, low cost, no battery requirement, long reading distance, and so forth. In this paper, two antennas at a certain distance apart are used to measure human walking speed by reading the time difference of the RFID tag. The received signal strength indicator (RSSI) of the RFID tag depends on the distances between the tag and the RFID reader. To calculate the walking speed, the time difference at RSSI maximum points and the discrete generalized cross-correlation algorithm are adopted to estimate the walking speed. The experimental results show that the precise measurement of the walking speed can be realized by employing the maximum time difference method and correlation time difference method. The former combines partitioned Gaussian fitting with Kalman filter, and the latter uses the Phat processor along with smoothed coherence transform (SCOT) weighting. These methods could be used to measure the daily walking speed of elderly individuals in senior health centers.

\section{Introduction}

The health of the elderly is a hotspot in recent scientific research. As population aging is intensifying, health care is being paid more attention. Researchers from The University of Pittsburgh drew a conclusion that the health of the elderly was closely associated with their walking speed. $^{(1)}$ The relationship between the health and walking speed of the elderly has also been studied and demonstrated by other researchers around the world. ${ }^{(2-6)}$ Measuring the walking speed of the elderly has high requirements for the system and algorithm. However, there is little research on human walking speed measurement and a practicable measuring method has not yet been established.

*Corresponding author: e-mail: 1fmo@seu.edu.cn http://dx.doi.org/10.18494/SAM.2018.1885 
Apart from that, almost all existing high-precision measuring instruments, such as laser Doppler velocimeter and radar speedometer, are designed for high-speed objects and are hard to popularize owing to their high cost. Moreover, in order to guarantee accuracy, speed measurement needs to be conducted without being noticed by the participants. However, most existing measuring instruments are easily noticeable, which causes poor accuracy of the results. To address this problem, UHF RFID tags, which are compact and low-cost, are used and discussed in this paper. The thickness of a tag is almost equal to that of a piece of paper, which makes it easy to be attached to a medical record card. Utilizing this kind of tag, the measurement can be carried out without being noticed by the participants. Furthermore, the maximum time difference and correlation time difference methods are adopted to calculate the walking speed. By analyzing and comparing the experimental results, it is shown that the methods have a good performance in measuring the walking speed.

\section{Mathematical Model of RFID Measurement}

\subsection{Space propagation loss model}

The signal propagation path loss model indicates the relation between signal propagation path loss and propagation distance. The shorter the distance between a tag and an antenna, the lower the loss during signal propagation. The equation of the signal propagation loss model in a vacuum can be expressed as Eq. (1). ${ }^{(7)}$

$$
P L=20 \lg (d)+20 \lg (f)+20 \lg \left(\frac{4 \pi}{c}\right)
$$

Here, $d$ is the distance between the tag and the antenna, $f$ is the operating frequency, and $c$ is the speed of light in a vacuum.

The equation can only be applied in a vacuum. In the real environment, there exist various propagation losses such as reflection, scattering, and absorption. Thus, for a real environment, the propagation loss model is usually described as the lognormal distribution model. ${ }^{(8)}$

$$
P_{r}(d)=P_{r}\left(d_{0}\right)-10 k \lg \left(\frac{d}{d_{0}}\right)-X_{\sigma}
$$

Here, $P_{r}(d)$ is the signal strength when the distance between the tag and the antenna is $d, d_{0}$ is the reference distance, and $k$ is path loss exponent. $X_{\sigma}$ is Gaussian random variable with zero means.

In a sample space with multiple wireless transmitters and receivers, the $P_{r}(d)$ of any single wireless transmitters and receivers follows a Gaussian distribution which can be described as $P_{r}(d) \sim\left(\overline{P_{r}(d)}, \sigma^{2}\right)$. As $X_{\sigma}$ is zero mean Gaussian random variable, $P_{r}(d)$ could be described as 


$$
P_{r}(d)=\overline{P_{r}(d)}-X_{\sigma}
$$

Combining Eqs. (2) and (3) gives

$$
\overline{P_{r}(d)}=P_{r}\left(d_{0}\right)-10 k \lg \left(\frac{d}{d_{0}}\right)
$$

According to Eq. (4), the relation between $d$ and $\overline{P_{r}(d)}$ can be denoted as

$$
d=d_{0} \times 10^{\frac{P_{r}\left(d_{0}\right)-\overline{P_{r}(d)}}{10 k}} .
$$

According to Eq. (5), the time when the distance between the tag and the antenna is the shortest can be determined by the maximum value of $\overline{P_{r}(d)}$, which is of great significance for calculating the speed.

\subsection{Speed calculation model}

As shown in Fig. 1, the reader receives signal strength $P_{r}(d)$ at different distances $d$ when a person with an RFID tag walks parallel to two antennas. The average walking speed can be calculated from the time it takes to walk past two antennas with known distance $D$ between the antennas. The equation is shown as

$$
v=\frac{D}{\Delta t}=\frac{D}{t\left(d_{\min 2}\right)-t\left(d_{\min 1}\right)} .
$$

Here, $D$ is the distance between two antennas, $t\left(d_{\min 1}\right)$ is the time when the tag is closest to antenna 1 , and $t\left(d_{\min 2}\right)$ is the time when the tag is closest to antenna 2.

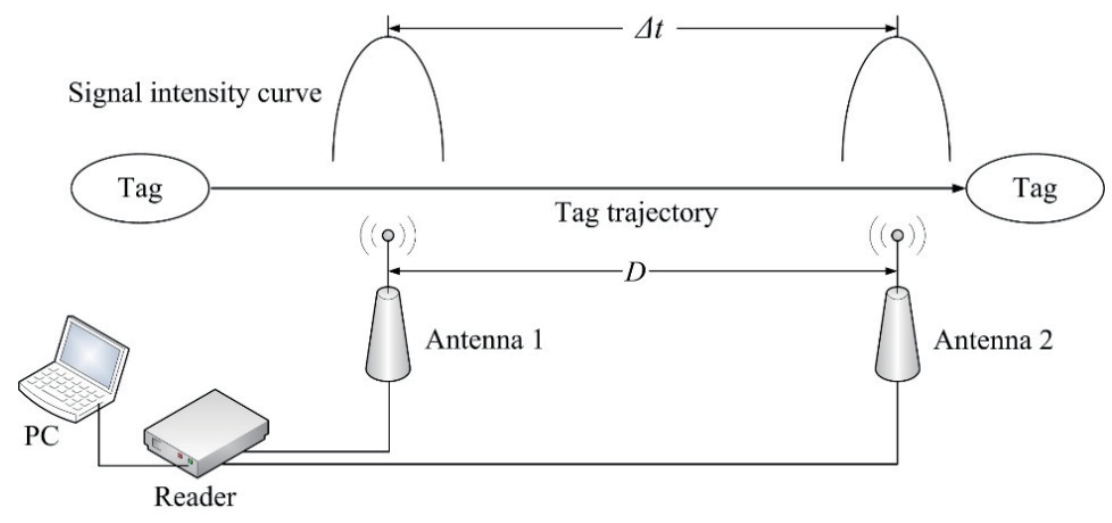

Fig. 1. (Color online) Construction of hardware. 


\section{Algorithm for RFID Speed Measurement}

Since the RFID reader receives data with non-constant time intervals and the signal strength is influenced by the multipath effect, the data have to be preprocessed. In order to generate a sequence with the time interval of $\Delta T$ and avoid the loss of the true value, the Lagrange interpolation is utilized to preprocess the data.

Assuming $\mathrm{P}, \mathrm{Q}$, and $\mathrm{H}$ are three adjacent points in the interpolation sequence with coordinates are $\left(x_{0}, y_{0}\right),\left(x_{1}, y_{1}\right),\left(x_{2}, y_{2}\right)$, respectively, and $x_{0}<x_{1}<x_{2}$, a parabola $y=a x^{2}+b x+c$ could be determined by these three points. The three parameters $a, b$, and $c$ can be calculated by the Lagrange interpolation. ${ }^{(9,10)}$

$$
y=\frac{\left(x-x_{1}\right)\left(x-x_{2}\right)}{\left(x_{0}-x_{1}\right)\left(x_{0}-x_{2}\right)} y_{0}+\frac{\left(x-x_{0}\right)\left(x-x_{2}\right)}{\left(x_{1}-x_{0}\right)\left(x_{1}-x_{2}\right)} y_{1}+\frac{\left(x-x_{0}\right)\left(x-x_{1}\right)}{\left(x_{2}-x_{0}\right)\left(x_{2}-x_{1}\right)} y_{2}
$$

After the calculation of three parameters $a, b$, and $c$, the interpolation will be implemented every $\Delta T$ in an interval $\left[x_{0}, x_{2}\right]$. Next, three new adjacent points will be chosen and the process will be repeated until the whole sequence is generated.

On the basis of interpolation, the maximum time difference method and correlation time difference method are applied to solve the walking speed.

\subsection{Maximum time difference method}

\subsubsection{Partitioned Gaussian fitting}

The signal strength $P_{r}(d)$ at a certain position can be described probabilistically. The more densely $P_{r}(d)$ is distributed, the more real $P_{r}(d)$ will be. Partitioned Gaussian fitting is applied to remove the bad data points from Lagrange interpolation sequence and find the $P_{r}(d)$ value with high probability. The equations are shown as below. ${ }^{(11,12)}$

$$
\left\{\begin{array}{l}
y=y_{0}+\frac{A}{\omega \sqrt{\frac{\pi}{2}}} e^{-2 \frac{\left(x-x_{c}\right)^{2}}{\omega^{2}}} \\
x_{c}=\frac{\sum_{i=1}^{i=k} P_{r}(d)_{i}}{k} \\
\omega=\sqrt{\frac{\sum_{i=1}^{i=k}\left(P_{r}(d)_{i}-x_{c}\right)^{2}}{k-1}}
\end{array}\right.
$$

Then, divide the Lagrange interpolation sequence into some intervals, each of which approximately represents a $P_{r}(d)$ value at a certain distance $d$. In the end, $P_{r}(d)$ of each interval is inserted into the fitting function and the high-probability data points which satisfy $0.5 \leq y \leq 1$ are retained. 


\subsubsection{Kalman filter}

Since partitioned Gaussian fitting can only cull bad data from Lagrange interpolation sequence, some high-probability noises still remain. The Kalman filter is adopted to process the data from Gaussian fitting, and reproduce the real state of the system by eliminating the effect of random interference. According to the features of the RFID system, a discrete Kalman filter can be applied to the data processing.

By taking the average value of $P_{r}(d)$ processed by Kalman filter, $\overline{P_{r}(d)}$ of the interval will be obtained. The time corresponding to $\overline{P_{r}(d)}$ is the average time $\bar{t}$ corresponding to $P_{r}(d)$.

\subsubsection{Speed calculation by maximum time difference method}

According to the monotony of Eq. (5), the calculation of $d_{\min 1}$ and $d_{\min 2}$ can be converted to the searching for the maximum of $\overline{P_{r}(d)}$. Then, the trouble of calculating path loss exponent $k$ could be avoided. $\bar{t}$ could be determined by the maximum of $\overline{P_{r}(d)}$, and Eq. (6) can be rewritten as follows.

$$
v=\frac{D}{\overline{t_{2}}-\overline{t_{1}}}
$$

\subsection{Correlation time difference method}

\subsubsection{Discrete generalized cross-correlation}

The cross-correlation function represents the degree of the dependence between two signal sequences. The greater the function value, the higher the dependence, and the better the crosscorrelation. When these two sequences are completely independent, the function value is zero. ${ }^{(14)}$ Thus, the cross-correlation function is of great significance in the calculation using two signal sequences.

Assuming that there are two signal sequences with time step $\Delta T$ interpolated by Lagrange interpolation:

$$
\begin{gathered}
y_{1}(n)=s(n)+m_{1}(n), \\
y_{2}(n)=s\left(n+n_{0}\right)+m_{2}(n) .
\end{gathered}
$$

Here, $s(n)$ is the useful signal received by antennas, $m_{1}(n)$ and $m_{2}(n)$ are the noises without crosscorrelation, and $n_{0}$ is the delay unit number of the signal cost from one antenna to the other.

Here is the cross-correlation function of $y_{1}(n)$ and $y_{2}(n)$ :

$$
R_{12}=E\left[y_{1}(n) y_{2}(n+m)\right] .
$$


Substitute Eqs. (10) and (11) into Eq. (12). Since $s(n), m_{1}(n)$, and $m_{2}(n)$ are uncorrelated, the value of cross-correlation between any two components will be zero:

$$
R_{12}=R_{s}\left(n_{0}-m\right)
$$

The cross-correlation function property shows that $R_{12}(m)$ will reach the maximum value when $m=n_{0}$. The delay $\tau$ between two signals is $n_{0} \Delta T$.

The calculation of a discrete cross-correlation sequence is divided into three parts, including shifting, multiplying, and summing. There is a linear correlation between two sequences $y_{1}(n)$ and $y_{2}(n)$. When the displacement of sequence $y_{2}(n)$ is $m$,

$$
R_{12}(m)=\sum_{n=-\infty}^{+\infty} y_{1}(n) y_{2}(n-m)
$$

When performing mutual correlation operation, zeros are put into the vacancies which appear because of shifting. At this time $y_{1}(n)$ and $y_{2}(n)$ are linearly related. Then, a conclusion could be drawn according to Cauchy-Schwarz inequality:

$$
\left|y_{1}(n), y_{2}(n)\right| \leq\left\|y_{1}(n)\right\|\left\|y_{2}(n)\right\| .
$$

The equal sign is only be taken when there is a linear correlation between $y_{1}(n)$ and $y_{2}(n)$. Now, the maximum cross-correlation calculation could be obtained. The product of displacement $m$ and the sampling period $\Delta T$ is the time difference between the two sequences.

The generalized cross-correlation algorithm is based on the cross-power spectrum between the two signals and it gives a certain weight in frequency-domain. ${ }^{(15,16)}$ The generalized crosscorrelation algorithm can whiten the signal and noise and enhance the high signal-to-noise ratio component, thus suppressing the influence of noise. And then the generalized cross-correlation function can be obtained by an inverse discrete fourier transform (IDFT) to the time domain:

$$
R_{12}^{g}(n)=I D F T\left[A(k) G_{12}(k)\right]
$$

Here, $G_{12}(k)$ is the cross-power spectral density between the two signals, and $A(k)$ is the generalized cross-correlation weighting function. ${ }^{(17)}$ The selection of weighting function depends upon different noise and reverberation conditions. Some commonly used weighting functions are shown in Table 1.

\subsubsection{Speed calculation by correlation time difference method}

Correlation between the frequency domain characteristics of two discrete signals can be described by power spectral density and cross power spectral density. The auto power spectral density and autocorrelation function could form a Fourier transform pair, and the cross power spectral density and cross-correlation function also could form a Fourier transform pair, ${ }^{(18)}$ giving 
Table 1

Commonly used weighting functions.

\begin{tabular}{lc}
\hline Name & Function expression \\
\hline Basic cross-correlation & $A(k)=1$ \\
Roth processor 1 & $A(k)=1 / G_{11}(k)$ \\
Roth processor 2 & $A(k)=1 / G_{22}(k)$ \\
Phat processor & $A(k)=1 /\left|G_{12}(k)\right|$ \\
SCOT & $A(k)=1 / \sqrt{G_{11}(k) G_{22}(k)}$ \\
\hline
\end{tabular}

$$
R_{12}(n)=\operatorname{IDFT}\left[G_{12}(k)\right] .
$$

According to the characteristics of the discrete time-domain circular convolution of the Fourier transform, Eq. (9) can be converted into Eq. (18):

$$
R_{12}^{g}(n)=A(n) \otimes R_{12}(n)=\sum_{m=0}^{N-1} A(m) R_{12 p}(n-m) R_{N}(n) .
$$

Here, $A(n)$ is obtained by $A(k)$ through discrete Fourier transform (DFT), $\otimes$ is circular convolution operator, $R_{12 p}(n-m) R_{N}(n)$ is a circumference shift sequence, and $R_{N}(n)$ is a rectangular sequence.

The matrix multiplication can be used to simplify the calculation of circular convolution. The matrix can be expressed as:

$$
\boldsymbol{Y}=\boldsymbol{H} \boldsymbol{X},
$$

where

$$
\begin{aligned}
& \boldsymbol{Y}=\left(\begin{array}{c}
R_{12}^{g}(0) \\
R_{12}^{g}(1) \\
R_{12}^{g}(2) \\
\vdots \\
R_{12}^{g}(N-1)
\end{array}\right) \\
& \boldsymbol{H}=\left(\begin{array}{ccccc}
R_{12}(0) & R_{12}(N-1) & R_{12}(N-2) & \ldots & R_{12}(1) \\
R_{12}(1) & R_{12}(0) & R_{12}(N-1) & \ldots & R_{12}(2) \\
R_{12}(2) & R_{12}(1) & R_{12}(0) & \ldots & R_{12}(3) \\
\vdots & \vdots & \vdots & \ddots & \vdots \\
R_{12}(N-1) & R_{12}(N-2) & R_{12}(N-3) & \ldots & R_{12}(0)
\end{array}\right) \\
& \boldsymbol{X}=\left(\begin{array}{c}
A(0) \\
A(1) \\
A(2) \\
\vdots \\
A(N-1)
\end{array}\right)
\end{aligned}
$$


$R_{12}^{g}(n)$ can be calculated by Eq. (14). The delay unit number between two signals can be determined by the maximum value of $R_{12}^{g}(n)$. The speed expression is presented as follows:

$$
v=\frac{D}{n_{0} \Delta T} .
$$

\section{Experimental Verification}

\subsection{Experiment design}

The Impinj R2000 UHF Reader module is adopted in the experiment. On the basis of Speedway Revolution's Autopilot technology, the reader operation in the application environment can automatically be optimized to ensure the best measurement performance. In the experiments, the distance $D$ between two antennas is set to 3,5, and $8 \mathrm{~m}$. As shown in Fig. 2, people carrying RFID tags walk along a straight line from one antenna to the other at different speeds. The real speed is calculated by the formula $D / \Delta t$, where $\Delta t$ is the travel time timed by the stopwatch. The data sequences of RSSI received are processed by means of the maximum time difference method and correlation time difference method. Furthermore, through comparison, the impact caused by different methods was analyzed. The performance of the algorithms is measured by the relative error defined as

$$
e=\frac{\left|v_{r}-v_{e}\right|}{v_{r}} \times 100(\%)
$$

Here, $v_{r}$ is the real walking speed, and $v_{e}$ is the estimated value of the walking speed.

\subsection{Single experimental result and analysis}

The first experiment was carried out in a walking corridor environment. The parameter $D$ is set to $8 \mathrm{~m}$ and the real speed is $1.1662 \mathrm{~m} / \mathrm{s}$. The experimental data was taken to analyze the error of the speed calculation algorithm.

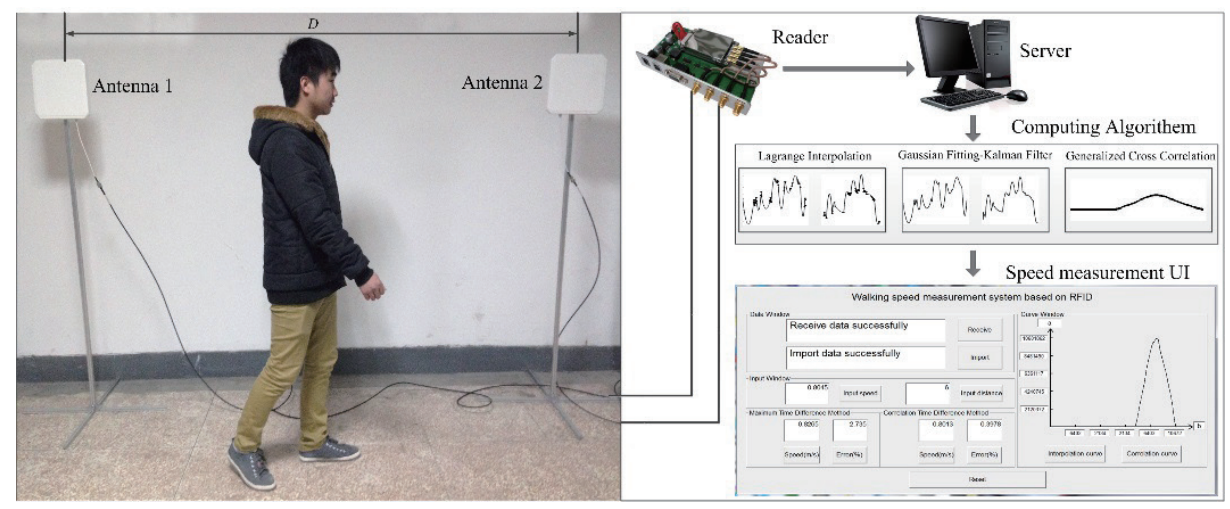

Fig. 2. (Color online) Hardware setup and algorithm. 


\subsubsection{Lagrange interpolation}

The original RSSI data are discrete points with an uneven distribution and are hard to process. To ensure an accurate value, the original data are interpolated by Lagrange interpolation to generate the sequence with a $0.001 \mathrm{~s}$ time interval. The results are presented in Fig. 3.

\subsubsection{Maximum time difference method}

The interpolated data are divided into several sections with an equal interval of $0.05 \mathrm{~s}$. Then, the divided data are fitted through the Gaussian fitting function. Meanwhile, the noises of the results are eliminated by the Kalman filter. As shown in Fig. 4, there is only a small change between the two curves. However, some small probability data and system random noise are culled, which makes the curve smoother and the data more reliable.

The average value of $P_{r}(d)$ is calculated by Gaussian fitting and Kalman filter as shown in Fig. 5. It is shown that the times corresponding to the maximum values of $\overline{P_{r}(d)}$ received by the two antennas are $3.148 \mathrm{~s}$ and $9.862 \mathrm{~s}$ respectively. According to Eq. (9), here is the calculation of speed $v$ :

$$
v=\frac{8}{9.862-3.148}=1.1915(\mathrm{~m} / \mathrm{s}) .
$$

\subsubsection{Correlation time difference method}

The data sequences with $\Delta T$ generated by Lagrange interpolation are corrected by four weighting functions respectively as shown in Fig. 6. As can be seen, the basic cross-correlation curve is relatively smooth, while the Roth processor has excessive noise. Although the curves of the Phat processor and smoothed coherence transform (SCOT) are not very smooth, the peaks

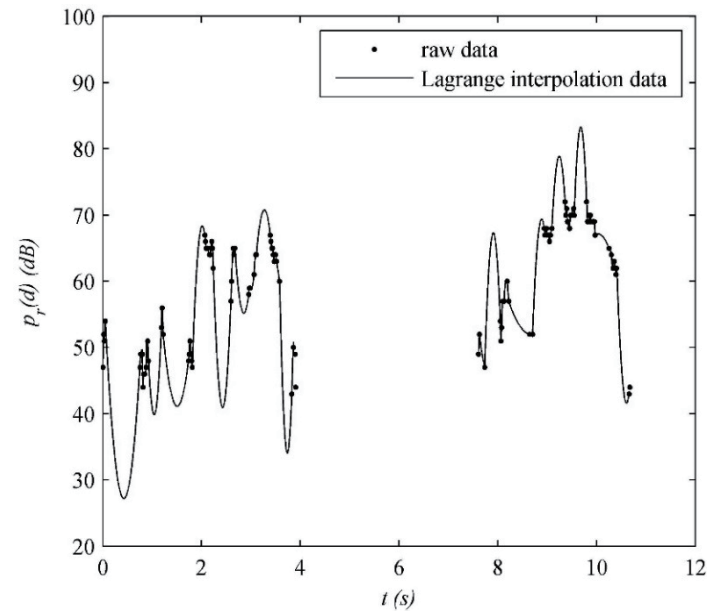

Fig. 3. Comparison of raw data and interpolated data.
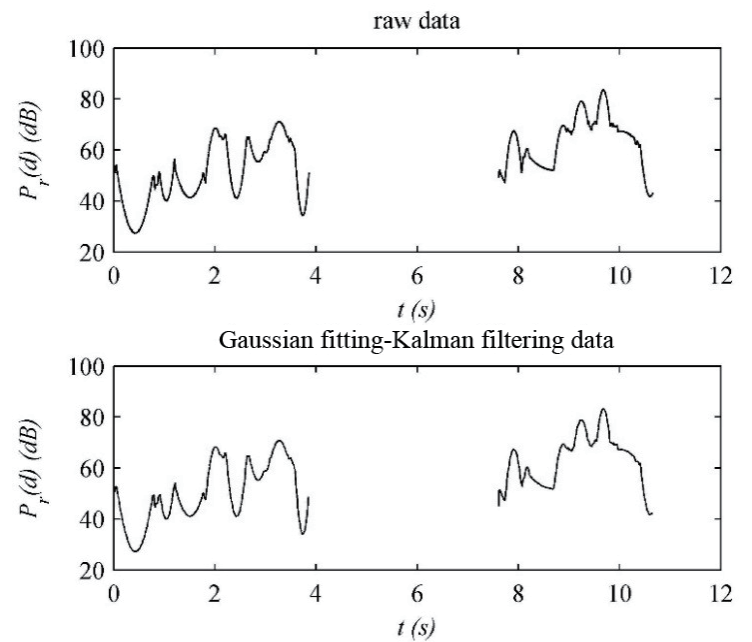

Fig. 4. Partitioned Gaussian fitting and Kalman filter process. 


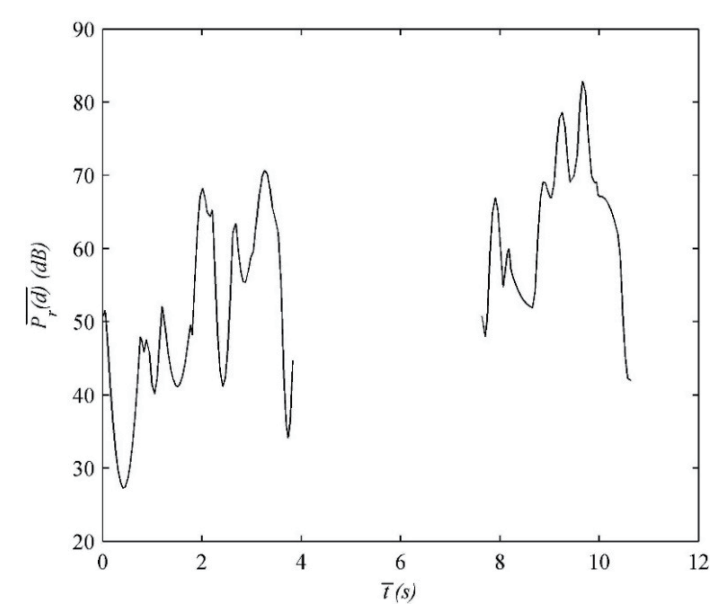

Fig. 5. Mean signal intensity curve.
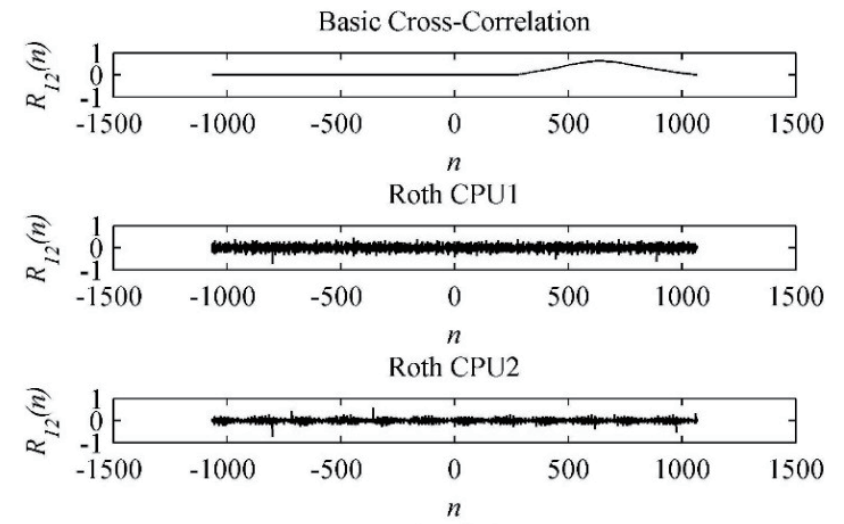

Phat CPU
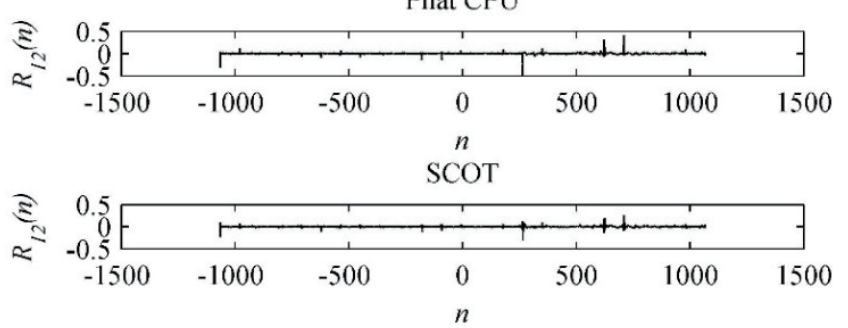

Fig. 6. Results processed by several common weighting functions.

are very clear and easy to distinguish, which makes it possible to measure the accurate delay of two signals in the actual measurement.

According to the calculation results of generalized cross-correlation and weighting functions processing, the delay unit number $n_{0}$ values are $41,-443,-357,689$, and 690 , respectively. The speed can be calculated by Eq. (20) and the results are 1.2480, $-1.8059,-2.2409,1.1611$, and 1.1594 $\mathrm{m} / \mathrm{s}$. In the light of the results of the Roth processor, it is easy to find that there is a huge error. So Roth processor function is inapplicable in this case while the others are relatively reasonable.

\subsubsection{Comparison of algorithms}

The speeds estimated by different methods are compared in Table 2. It can be seen from the table that the accuracy of the two calculation methods is relatively high. In order to ensure a higher authenticity of the preserved data, Gaussian fitting is used to remove the data with low probability. According to the space propagation loss model, the distance can be determined by the mean value of the signal intensity $\overline{P_{r}(d)}$. It can reduce the random error and achieve a higher accuracy than the conventional polynomial fitting. The conventional cross-correlation algorithm cannot suppress the effects of the noise, which can cause a relatively large error when calculating the delay. However, the noise can be suppressed by generalized cross-correlation function derived by the inverse Fourier transform. The peaks of Phat processor and SCOT are prominent, which means that the impact of the noise is suppressed effectively. The data processed by the basic cross-correlation are relatively smooth, while the peak value is not so 
Table 2

Results of maximum time difference method and correlation time difference method.

\begin{tabular}{lcc}
\hline Calculation method & Speed $(\mathrm{m} / \mathrm{s})$ & Error $(\%)$ \\
\hline Maximum time difference method & 1.1915 & 2.169 \\
Basic cross-correlation & 1.2480 & 7.014 \\
Roth processor 1 & -1.8059 & Unreasonable \\
Roth processor 2 & -2.2409 & Unreasonable \\
Phat processor & 1.1611 & 0.437 \\
SCOT & 1.1594 & 0.583 \\
\hline
\end{tabular}

clear, which could easily lead to errors. The Roth processor has excessive noises, which means the results of the Roth processor are unreasonable.

\subsection{Multiple experimental results and analysis}

For further analysis, multiple experiments with different waking speeds and different antenna distances were carried out in a room space environment. Errors of different algorithms and errors of different antenna distances at different waking speeds were analyzed respectively.

\subsubsection{Errors of different algorithms at different walking speeds}

The experimental data whose $D$ is set to $5 \mathrm{~m}$ is taken to illustrate the results as shown in Fig. 7. It can be seen that correlation time difference method using the Phat processor and SCOT weighting is more accurate than the maximum time difference method which combines partitioned Gaussian fitting with Kalman filter. The algorithm errors of the correlation time difference method are less than $1 \%$. The main reason is that every data point is considered by the correlation time difference method, while the maximum time difference method only considers the maximum point.

\subsubsection{Algorithm errors at different distance $D$}

Signal interference between the antennas changes with the distance $D$, and it can cause the measurement error. The results of the correlation time difference method using the SCOT weighting at different distance $D$ are illustrated in Fig. 8. According to Fig. 8, the correlation time difference method using the SCOT weighting can maintain a relatively high accuracy at different distance $D$ and it can reduce the effect of the distance $D$.

The error of the correlation time difference method used in the manuscript is less than $1 \%$, i.e., for the usual walking speed of the elderly, the accuracy can reach $0.02 \mathrm{~m} / \mathrm{s}$. In the previous research of Studenski et al., it was found that the walking speed causes a difference with increments of $0.1 \mathrm{~m} / \mathrm{s} .{ }^{(1)}$ Therefore, this method is accurate enough for the health assessment of the elderly.

\section{Conclusions}




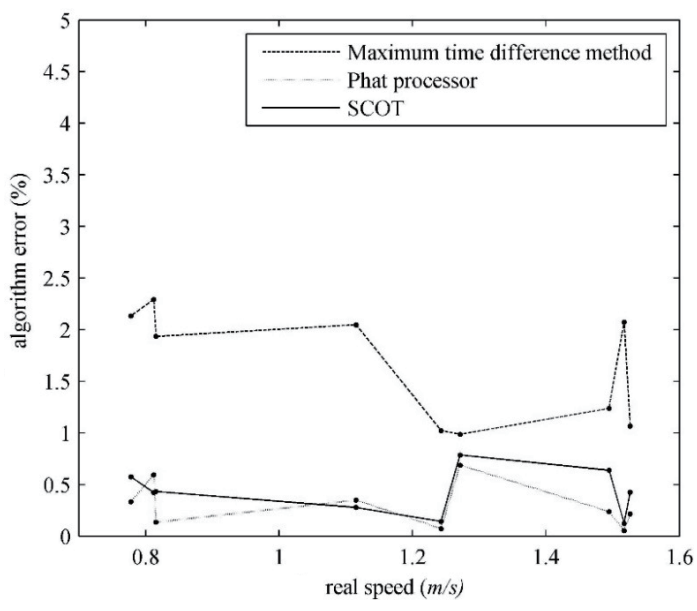

Fig. 7. Algorithm errors at different walking speeds.

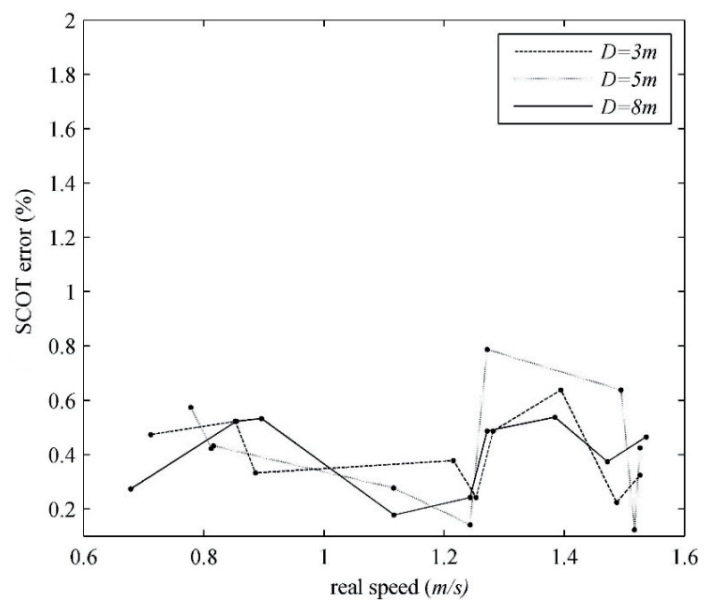

Fig. 8. SCOT errors at different distance $D$.

Walking speed measurement methods based on UHF RFID are analyzed in this paper, and the walking speed of the participant is calculated by the means of maximum time difference and correlation time difference. The maximum time difference method combines partitioned Gaussian fitting with Kalman filter, and the correlation time difference method utilizes the Phat processor along with SCOT weighting. Through the comparison of experimental results, a conclusion can be drawn that the two methods can effectively reduce errors and have good performance in estimating the walking speed, which can be applied to the health assessment of the elderly. In daily life, however, a walking path is not a straight line, in which case a third antenna could be used to determine the motion in a plane and calculate the walking speed using pairwise cross-correlations.

\section{Acknowledgments}

This work was supported by the National Science Foundation of China (61603091, MultiDimensions Based Physical Activity Assessment for the Human Daily Life).

\section{References}

1 S. Studenski, S. Perera, K. Patel, C. Rosano, K. Faulkner, M. Inzitari, J. Brach, J. Chandler, P. Cawthon, E. B. Connor, M. Nevitt, M. Visser, S. Kritchevsky, S. Badinelli, T. Harris, A. B. Newman, J. Cauley, L. Ferrucci, and J. Guralnik: JAMA 305 (2011) 1.

2 S. Shinkai, S. Watanabe, S. Kumagai, Y. Fujiwara, H. Amano, H. Yoshida, T. Ishizaki, H. Yukawa, T. Suzuki, and H. Shibata: Age. Ageing 29 (2000) 5.

3 M. Montero-Odasso, M. Schapira, E. R. Soriano, M. Varela, R. Kaplan, L. A. Camera, and L. M. Mayorga: J. Gerontol. A-biol. 60A (2005) 10.

4 N. Jitramontree: J. Gerontol. Nurs. 36 (2010) 11.

5 T. L. Schwenk: Massachusetts Med. Soc. 31 (2011) 2.

6 F. Garcia-Pinillos, M. Cozar-Barba, M. Munoz-Jimenez M, V. Soto-Hermos, and P. Latorre-Roman: 
Psychogeriatrics 16 (2016) 3.

7 M. Hasani, E. S. Lohan, L. Sydänheimo, and L. Ukkonen: Proc. 2014 IEEE RFID Technology and Applications Conf. (IEEE, 2014) 170-174.

8 H. Ma and K. Wang: IEEE Sens. J. 17 (2017) 11.

9 A. Shahsavaran and A. Shahsavaran: Appl. Math. Sci. 6 (2012) 18.

10 P. O. J. Scherer: Interpolation (Springer, Berlin, Heidelberg, 2010) p. 15.

11 F. Seco, C. Plagemann, A. R. Jiménez, and W. Burgard: Proc. 2010 IEEE Indoor Positioning and Indoor Navigation Conf. (IEEE, 2010) 1-8.

12 J. Zhan, L. X Wu, and Z. J Tang: Telecommun. Eng. 50 (2010) 4.

13 R. Kalman: J. Basic Eng. 82 (1960) 1.

14 X. L. He, L. Z Yang, and X. S Yang: Prog. Geophysic. 24 (2009) 4.

15 C. H. Knapp and G. C. Carter: IEEE Trans. ASSP 24 (1976) 4.

16 Y. Gao, Y. Chen, and A. Bekkali: IEEE Commun. Lett. 20 (2016) 4

17 J. D. Chen, J. Benesty, and Y. T. Huang: EURASIP J. Adv. Sig. Process. 2005 (2005) 1.

18 W. A. Grardener: IEEE Commun. Lett. 40 (1992) 1. 